\title{
CUBIERTA HINCHABLE DE LA PLAZA DE TOROS DE VALLADOLID. UN INTENTO FRUSTRADO
}

\author{
(A PNEUMATIC DOME ON THE "ARENA" OF VALLADOLID. A FAILED ATTEMPT)
}

Juan Monjo Carrió, Dr. Arquitecto. ETSAM

Antonio Blázquez Morales, Arquitecto. ICCET/CSIC

Antonia Pacios Alvarez, Arquitecto.

ESPAÑA

Fecha de recepción: $10 \cdot$ VII-90

\section{RESUMEN}

El presente articulo contiene los cálculos previos y trabajos preparatorios para el cerramiento del ruedo de la plaza de toros de Valladolid mediante una cubierta hinchable en forma de casquete esférico, ejecutada con lámina de polietileno y refuerzos radiales de cinta de perlón.

Dicha cubierta tenia el objetivo de albergar temporalmente (10 dias) la exposición de materiales y sistemas constructivos (CONSTRUVALL-89) organizada por los alumnos de $6 .^{\circ}$ curso de la Escuela Técnica Superior de Arquitectura de la Universidad de Valladolid, en marzo de 1989.

Se describen, asimismo, los trabajos de confección y montaje de la cubierta, asi como el intento de hinchado de la misma que, desgraciadamente, resultó frustrado, debido, sobre todo, a las malas condiciones atmosféricas del momento.

\section{SUMMARY}

This article contains the previous calculations and preparatory works for the covering of the Arena of Valladolid with a penumatic dome in the shape of a segment of a sphere, made out of polietilene membrane and radial "perlon" ribbons reinforcement.

The objective of the dome being the temporary covering (10 days) of the Building Techniques and Materials Show (CONSTRUVALL-89) organized by the School of Architecture students at the University of Valladolid in March 1989.

The works for the making up of the dome are also described as well as the attempt for its inflation that, unfortunately, failed due, above all, to the bad weather conditions of those days.

\section{INTRODUCCIÓN}

Este trabajo recoge el seguimiento y análisis de la ex. periencia de los alumnos de $6 .^{\circ}$ curso de la Escuela T. S. de Arquitectura de Valladolid en su intento de cubrir la plaza de toros de la ciudad para albergar a los expositores de la feria de Construcción, Diseño y Tec. nologia -CONSTRUVALL-89- prevista entre los dias 27 de abril y 4 de marzo de 1989.

El antecedente más directo de este tipo de experiencias fue realizado también en Valladolid e igualmente por alumnos de último curso de carrera, en el año 1985, y consistió en el cubrimiento de una pista polideportiva con unos medios y sistemas muy similares a los planteados en este proyecto.

Desgraciadamente, y a pesar del esfuerzo y la ilusión puestas en ello, el intento no ha conseguido el éxito deseado por multitud de circunstancias, especialmente climáticas, que se comentan en el último punto de este artículo.

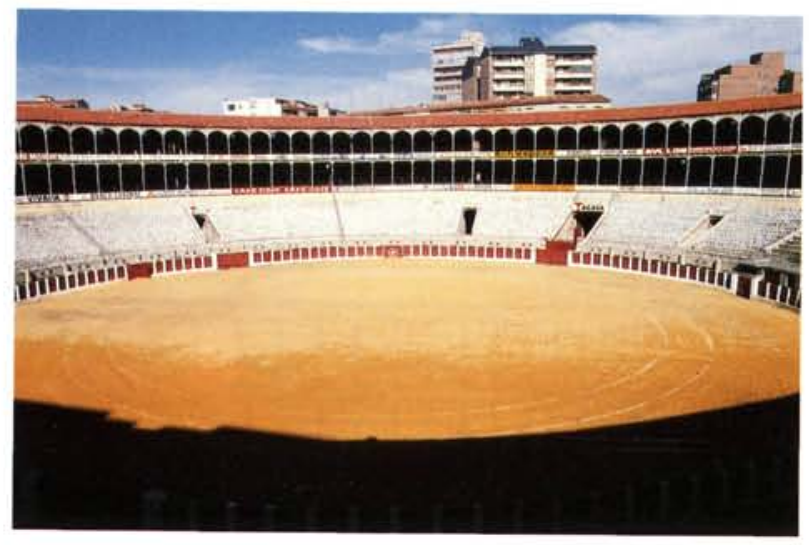

Plaza de Toros de Valladolid. España. 


\section{PLANTEAMIENTO}

La planta de la plaza de toros que se deseaba cubrir tiene un diámetro total de $56,45 \mathrm{~m}$ incluído el callejón, lo que da una superficie en planta posible de $3.544 \mathrm{~m}^{2}$ (Fig. 1).

El interés por cubrir esta plaza sin soportes intermedios, y el deseo de un ambiente interno controlado, encaminaron hacia la elección de una estructura neumática, soportada por aire, que tiene como principales ventajas, además de las indicadas, la luminosidad y movilidad.

En general, las aplicaciones de las estructuras neumáticas se realizan con membranas de escasa elasticidad por lo que la forma final, inflada, debe generarse a partir del diseño más aprovechable de los patrones, teniendo en cuenta que ante una membrana elástica la presión de hinchado tiende a dar una forma esférica, por ser ésta la que encierra más volumen con la menor superficie, al igual que ocurre con las pompas de jabón.

En cualquier caso, una vez decidida la cubierta de este tipo, es necesario definir sus características generales.

En primer lugar, el tipo de presión. En este caso, al ser "soportada por aire", baja presión, valores entre 10 a $100 \mathrm{~kg} / \mathrm{m}^{2}$.

En segundo lugar, el tipo de membrana. Se eligió lámina simple de polietileno, de 800 galgas.

En tercer lugar, se decidió usar un radio de curvatura que redujese la acción del viento a succión que, en cualquier caso, colabora en la estabilidad de la cubierta al introducir tracciones, permitiendo disminuir la sobrepresión interior con el consiguiente ahorro.

Esta última decisión, obligó a adoptar la incorporación de un soporte lineal radial en la membrana, tipo cable, que, como veremos posteriormente, consistió en una cinta de perlón de alta resistencia.

Una vez colocada la membrana y para impedir fugas de aire, se colocarán faldones desde la barandilla has. ta el suelo.

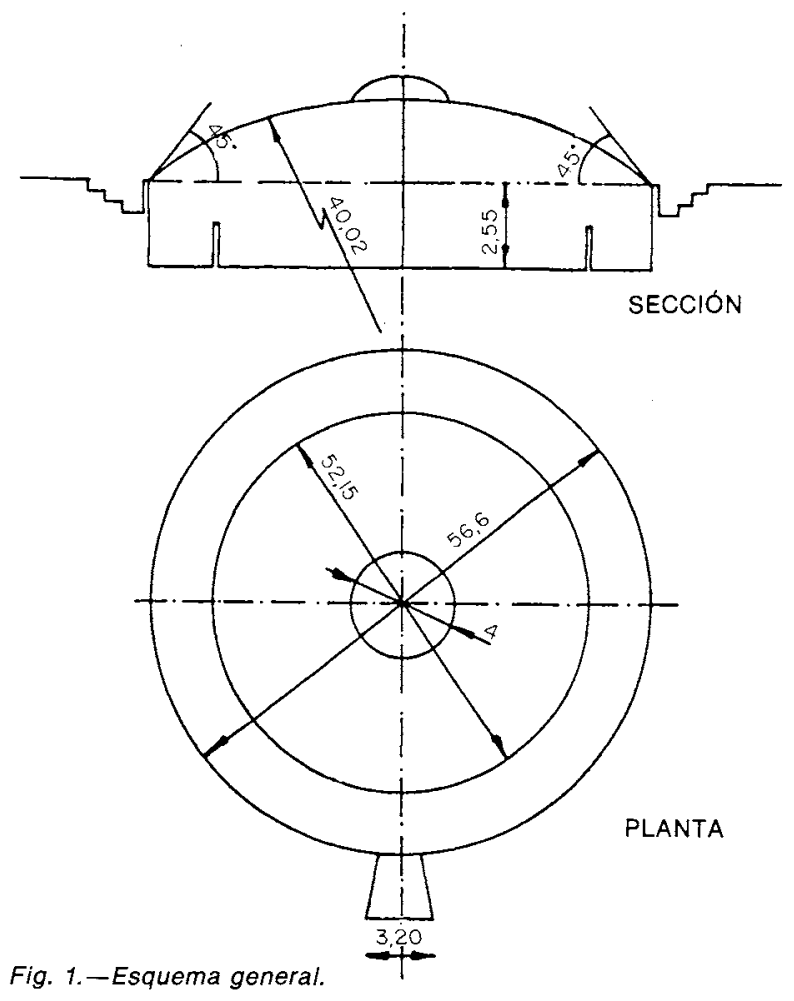

Esta cinta reduce la tensión en la membrana al disminuir su radio de curvatura transversal y absorbe los incrementos de esfuerzos en caso de mayor carga de viento.

Por último, la tensión de tracción que se produce en la membrana es transmitida a los bordes por medio de su anclaje, que en este caso debería ser la barandilla de cable de acero que recorre la barrera de la plaza, anclada según puede verse en la foto 1. Como refuerzo de esta barandilla se incorpora una red de tornapuntas, formando una triangulación de la misma (foto 2). El aspecto previsto interiormente está reflejado en el dibujo realizado por uno de los alumnos (Fig. 2).

Igualmente deberá resolverse la estanquidad del acceso. Para ello, se dispone una doble puerta de estructura rígida, cubierta con plástico, que sirve igualmente de control de entrada.

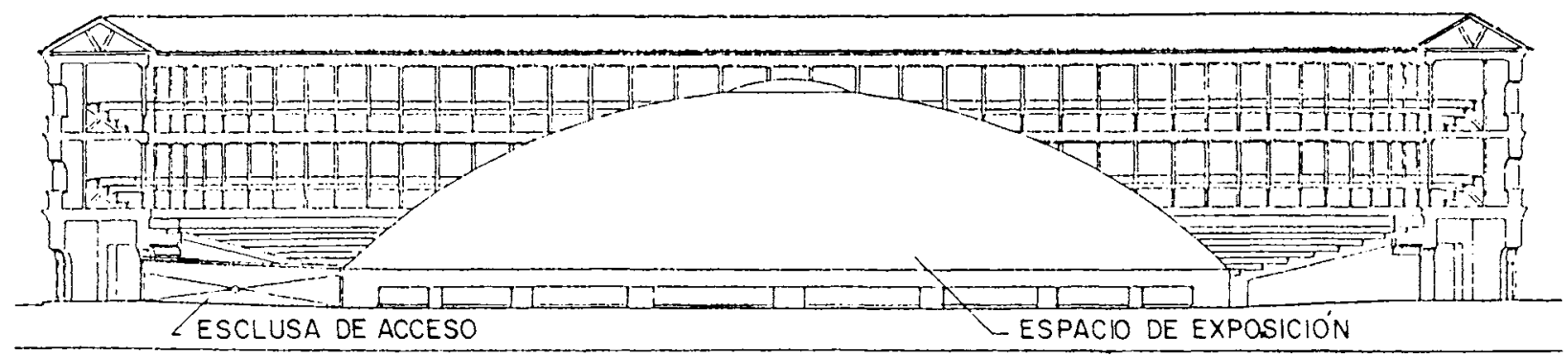




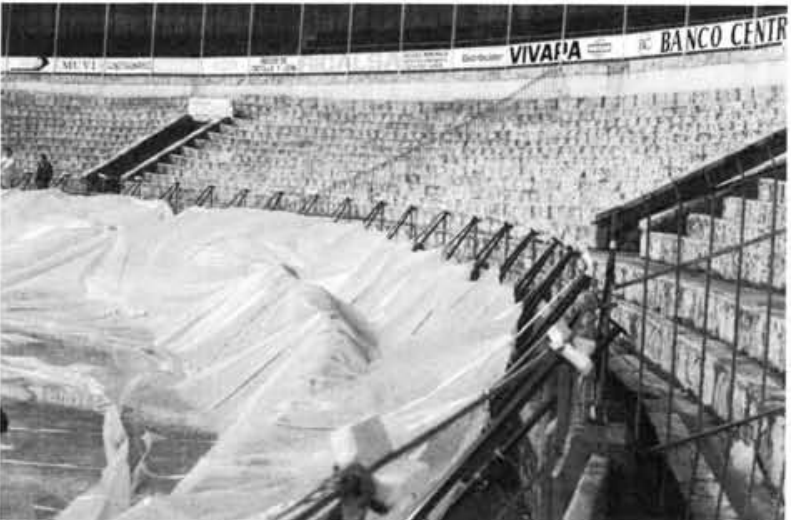

Foto 1

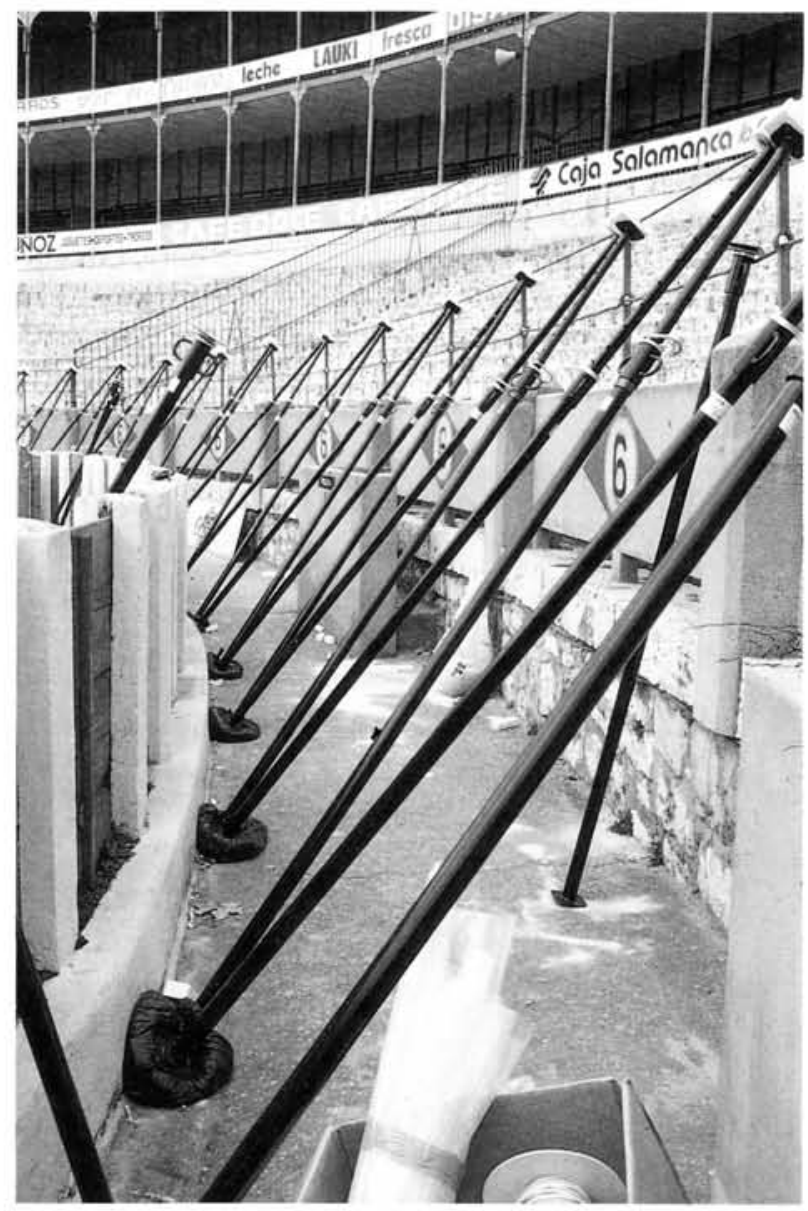

Foto 2 a)

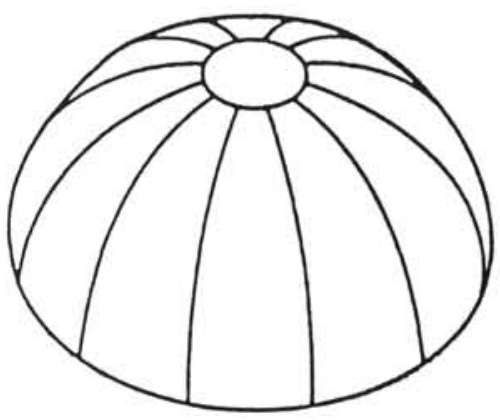

b)
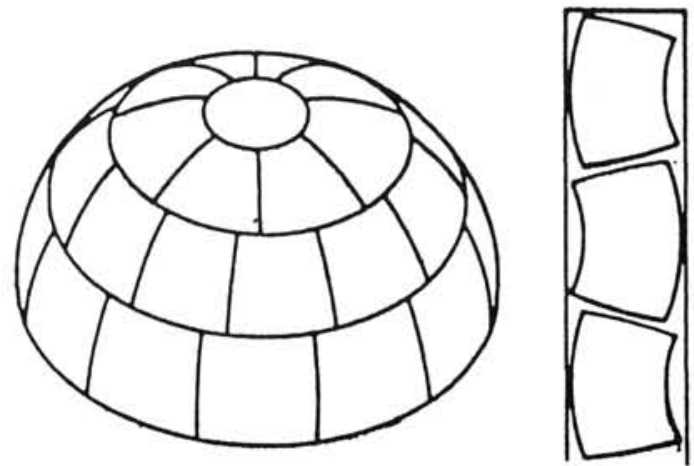

Fig. 3.-Patrones posibles.

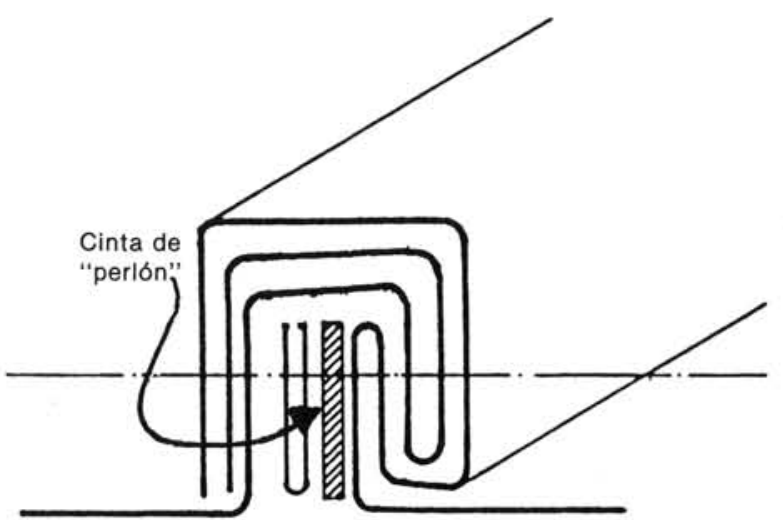

Fig. 4.-Solución de junta grapada con refuerzo de cinta.
Resueltas todas estas caracteristicas generales quedaban por definir los siguientes aspectos concretos.

Primero, los patrones de corte, que debian ser elegidos entre las opciones "a" y "b" de la figura 3.

Como la opción "b" es más costosa, aunque distribuya mejor las costuras sobre la totalidad de la superficie, se eligió la opción "a", a pesar de la desventaja (c) Consejo Superior de Investigaciones Científicas Licencia Creative Commons 3.0 España (by-nc) que supone el incremento de las costuras hacia la parte superior de la cúpula.

Segundo, el tipo de junta. Ėsta debía ser sencilla de ejecución, por lo que se eligió la unión con grapas metálicas con los pliegues que pueden verse en la fig. 4.

Tercero, los dispositivos de estabilización. Esta estabilización se hace mediante equipos de inflado dispuestos en el exterior de la estructura. 
La determinación de la capacidad que se requerirá a los ventiladores depende, tanto de las características de éstos, como de la estanquidad general de la estructura. Las fluctuaciones circunstanciales que se produzcan en la presión del soporte pueden ser peligrosas con presencia de nieve o de cargas de viento. Por ello deberá preverse la posibilidad de aumentar la presión interna para consolidar la membrana en su posición.

Igualmente, estos ventiladores tendrán la función de renovar el aire; del orden de 1 a 2 cambios totales de aire por hora.

\section{MATERIALES}

Los materiales empleados fueron los siguientes:

Plástico. Lámina de polietileno de 2 metros de ancho, color blanco y espesor 800 galgas $(0,2 \mathrm{~mm})$ cedido gratuitamente por Repsol.

La resistencia teórica de este plástico debia ser de $300 \mathrm{~kg} / \mathrm{m}$.

Este material, es aceptable para una construcción de este tipo, por su trabajabilidad y flexibilidad, además

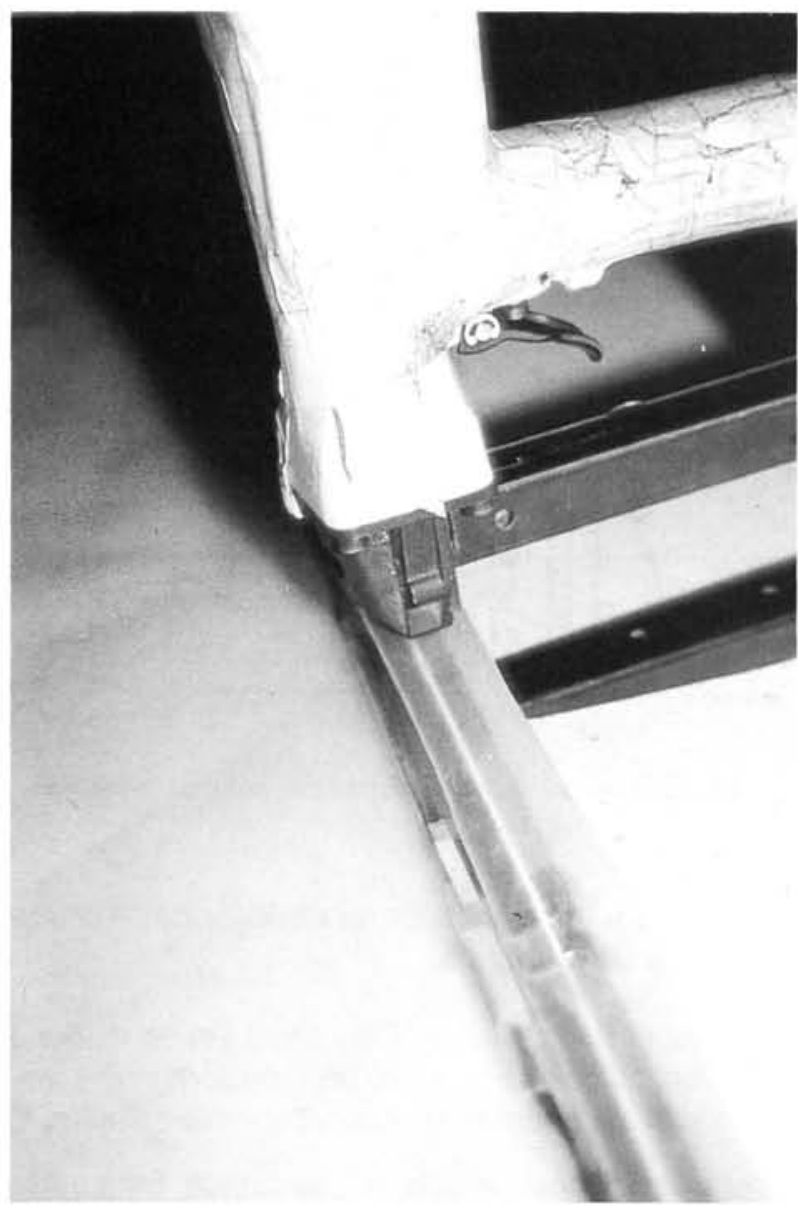

Foto 3 de que en este caso resultó excepcionalmente económico.

Se hicieron ensayos sobre la resistencia real a tracción del plástico, al tiempo que se ensayaban las juntas, sin que apareciesen problemas.

Grapas. Las grapas para el cosido de las diferentes láminas son de tipo industrial de acero protegido contra la corrosión aplicadas con ayuda de compresor. Este sistema de unión es evidentemente temporal, como era la idea en sí del proyecto (fotos 3 y 3').

Se colocaron cada $5 \mathrm{~cm}$ y el número total de grapas colocadas superó las 300.000 .

Refuerzo. Como complemento estructural se introdujo en las uniones entre patrones una cinta de PERLÓN de alta resistencia (hasta los $1.500 \mathrm{kp}$ ), de las habitualmente utilizadas en alpinismo (fotos 4 y $4^{\prime}$ ).

Ventiladores. Se emplearon 6 ventiladores centrifugos de potencia total $21 \mathrm{~kW}$, repartidos a lo largo del perimetro. Parte de estos ventiladores se utilizarian para mantenimiento y todos ellos para el levantamiento de la estructura.

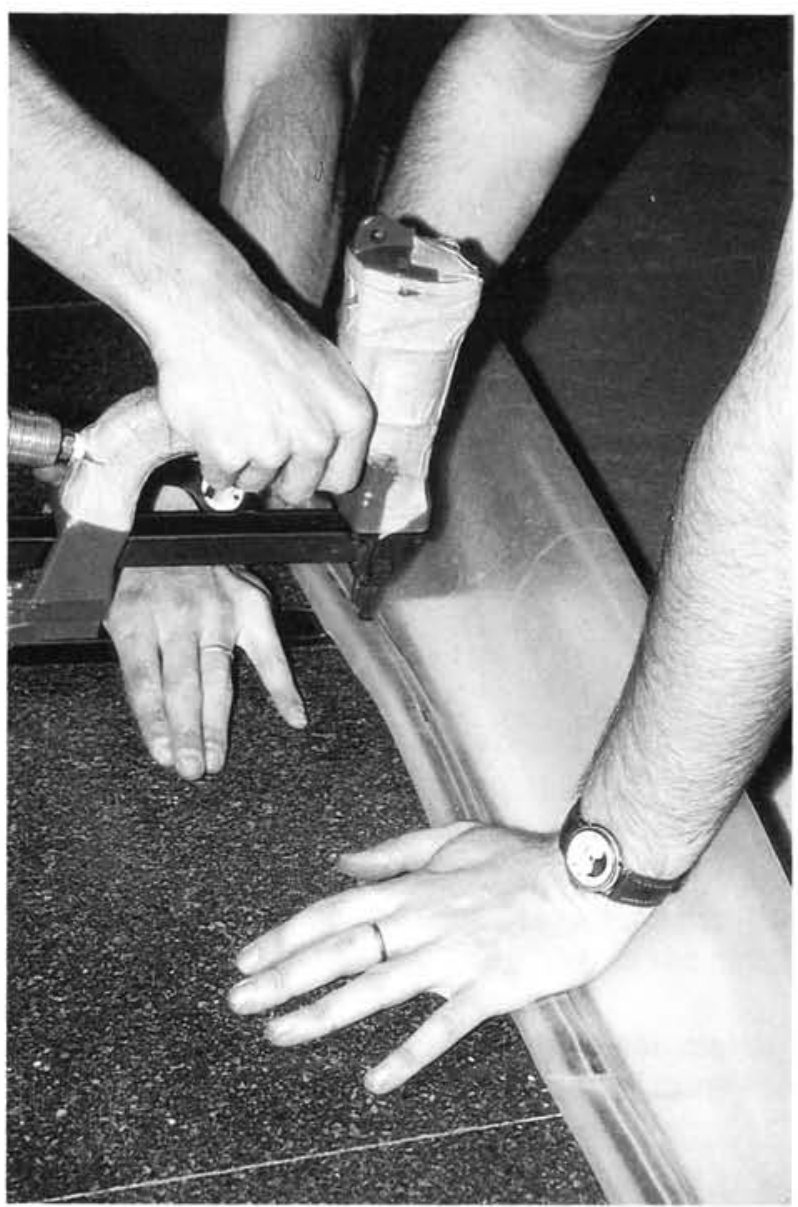

Foto 3 


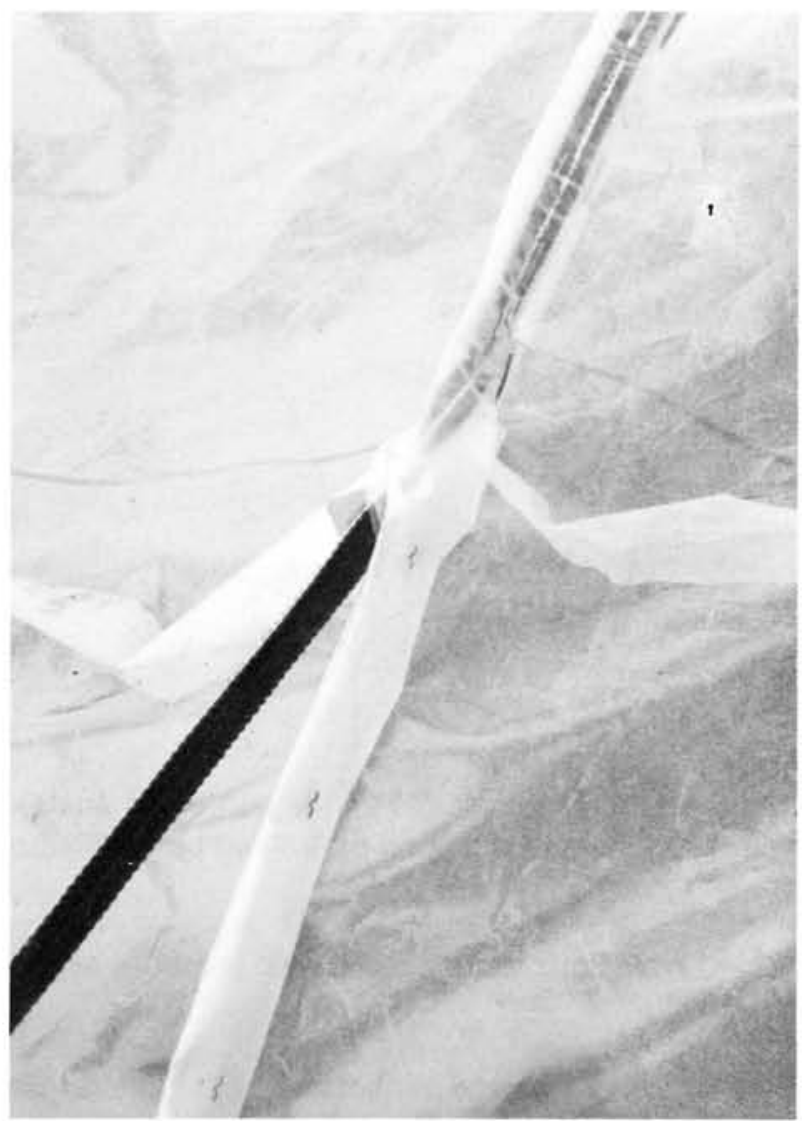

Foto 4

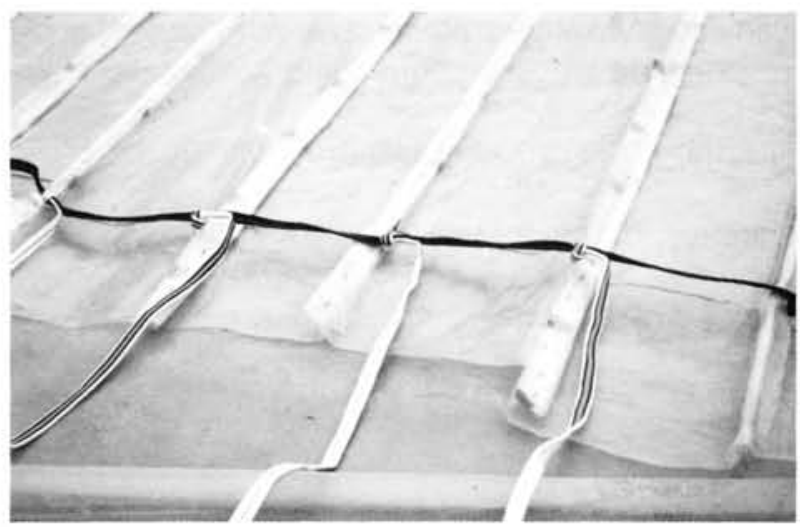

Foto $4^{\prime}$

\section{CÁLCULO}

El sistema de cálculo empleado para éste tipo de cubiertas resuelve dos problemas fundamentalmente.

Por un lado, la membrana debe resistir la tensión tangencial que en ella se produce como consecuencia de la presión de aire interior, así como los esfuerzos acumulados en las uniones de patrones.

Además, el anclaje debe garantizar la estabilidad de la cubierta.

(c) Consejo Superior de Investigaciones Científicas Licencia Creative Commons 3.0 España (by-nc)

\subsection{Cálculo general}

Adoptando la normativa alemana, necesitamos una presión interior de $30 \mathrm{~kg} / \mathrm{m}^{2}$ (para alturas superiores a $8 \mathrm{~m}$ ) que mantenga la membrana elevada. Esta presión y el radio de curvatura nos definen la tensión tangencial a la que trabajará la tela, en la relación:

$\sigma_{1}=\mathrm{P}_{1} \cdot \mathrm{R} / 2$

Según el esquema adjunto,

$$
\begin{aligned}
& R^{\prime 2}=2 R^{2} \text { o bien } R=R^{\prime 2} / 2 \\
& \underline{R}=40,02 m \\
& h=28,3 \cdot \operatorname{tg} 22,5=11,72 m \\
& \underline{H}=14,27 m=11,72+2,55
\end{aligned}
$$

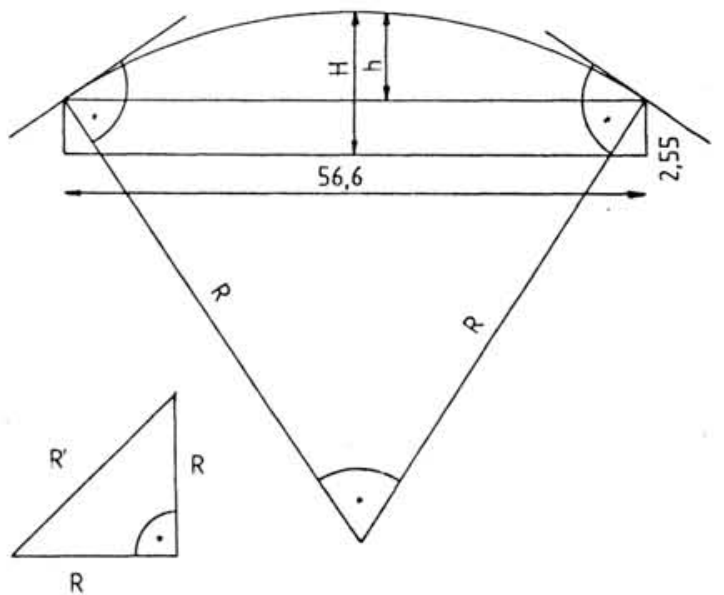

Con el valor de la presión mínima por normativa, la tensión en la membrana sería:

$$
\sigma_{1}=30 \mathrm{~kg} / \mathrm{m}^{2} \cdot 40,02 \mathrm{~m} / 2=600,8 \mathrm{~kg} / \mathrm{m} \text {. }
$$

Considerando que la tensión tangencial obtenida es elevada se dota a la cubierta de una segunda curvatura por cada módulo patrón, normal a la de los meridianos, cuyo radio máximo $r_{\text {max }}$ se puede obtener de la siguiente forma:

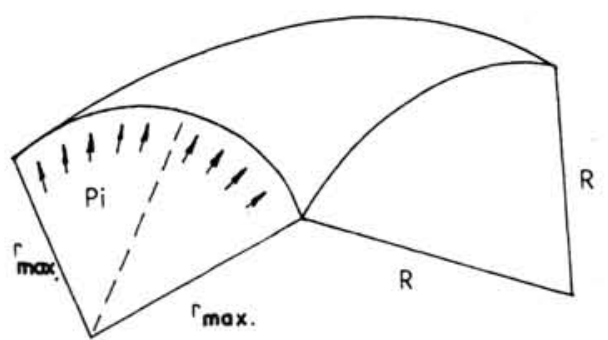


Al tener distintas curvaturas en cada punto,

$P_{1}=\sigma_{1} \cdot(1 / R+1 / r)$

$\sigma_{1 \text { max }}$ admitida por la membrana es de $300 \mathrm{~kg} / \mathrm{m}^{2}=$ $=(15 \mathrm{~kg} / 5 \mathrm{~cm})$.

$30 \mathrm{~kg} / \mathrm{m}^{2}=300 \mathrm{~kg} / \mathrm{m} \cdot(1 / 40,02+1 / \mathrm{r})$

$r_{\text {max. }}, 13,33 \mathrm{~m}$, se eligió $r=10 \mathrm{~m}$

que es menor que el $r_{\max }$

Con estas condiciones geométricas, la tensión máxima de trabajo es:

$30 \mathrm{~kg} / \mathrm{m}^{2}=\delta_{t} \cdot(1 / 40,2+1 / 10)=240,24 \mathrm{~kg} / \mathrm{m}$.

$\delta_{\mathrm{t}}=240,24 \mathrm{~kg} / \mathrm{m}$ que equivale a $12,012 \mathrm{~kg} / 5 \mathrm{~cm}$, inferior a la resistida por la membrana de polietileno elegida.

\subsection{Cálculo del casquete}

Para que la tela esté a la misma tensión, el radio de curvatura debe ser de $16 \mathrm{~m}$ y la altura del casquete de $0,50 \mathrm{~m}$. Por razones estéticas esta altura se consideró insuficiente, por lo que se fue a un radio menor, aunque eso implicase que la tela estuviese menos tensa.

Si reducimos la tensión hasta $8 \mathrm{~kg} / 5 \mathrm{~cm}$ que es aproximadamente $160 \mathrm{~kg} / \mathrm{m}$

$30 \mathrm{~kg} / \mathrm{m}^{2}=160 \mathrm{~kg} / \mathrm{m} \cdot(2 / \mathrm{r})$

$r=10,67 \mathrm{~m}$ que es aproximadamente $10 \mathrm{~m}$

$d=R^{2} \cdot 4^{2}=9,892 \mathrm{~m}$

$\underline{h}=r \cdot d=10,67 \cdot 9,89=\underline{0,77 m}$

más aceptable, estéticamente hablando.

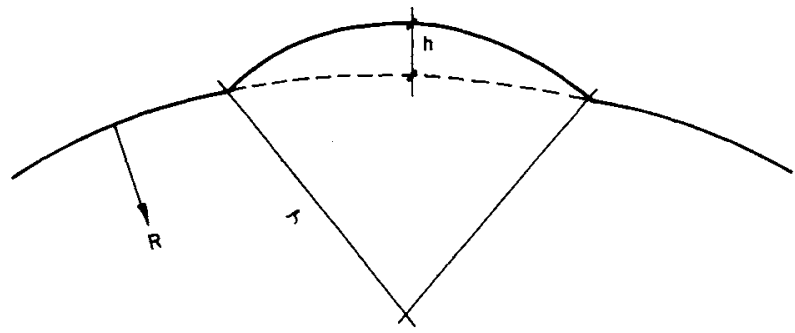

Para esta curvatura la $\sigma_{\text {trab }}=7,5 \mathrm{~kg} / 5 \mathrm{~cm}$ que equivale a $\sigma_{\text {trab }}=150 \mathrm{~kg} / \mathrm{m}$.

\subsection{Acceso}

Por sus reducidas dimensiones, para la presión admisible de $30 \mathrm{~kg} / \mathrm{m}^{2}$ la tensión es pequeña.
Al tener forma cilindrica, $P_{1}=\sigma_{1} / \tau$

$30 \mathrm{~kg} / \mathrm{m}_{2}=\sigma_{1} \cdot\left(1 / \mathrm{r}_{1}\right)=\sigma_{1} \cdot(1 / 1,5 \mathrm{~m})$

$\sigma_{1}=2,25 \mathrm{~kg} / 5 \mathrm{~cm}$.

\subsection{Anclaje}

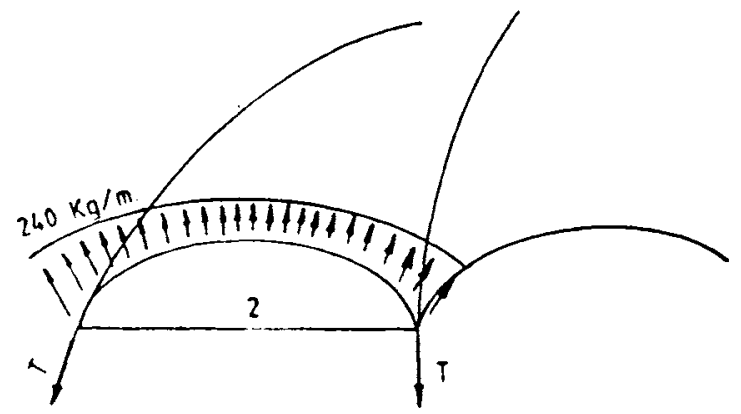

Las tensiones acumuladas en los puntos de anclaje son resultado de la tensión de trabajo por la distancia entre ellos.

$\mathrm{T}=240 \mathrm{~kg} / \mathrm{m} \cdot 2 \mathrm{~m}=480 \mathrm{~kg} ; \mathrm{T} \cdot=960 \mathrm{kp}$ (coef. de seguri$\mathrm{dad}=2$ ).

Esta tensión la absorbe perfectamente la cinta de perlón, que se usa como refuerzo en las juntas y pasa directamente a los anclajes.

Considerando el peso propio de la estructura de la plaza existente en cada punto como contrapeso,

pilastra, $\quad 0,30 \mathrm{~m} \cdot 0,30 \mathrm{~m} \cdot 1,00 \mathrm{~m} \cdot 2,4 \mathrm{t} / \mathrm{m}^{3}=0,216 \mathrm{t}$

murete, $\quad 0,09 \mathrm{~m} \cdot 0,73 \mathrm{~m} \cdot 1,80 \mathrm{~m} \cdot 2,4 \mathrm{t} / \mathrm{m}^{3}=0,283 \mathrm{t}$

base, $\quad 2 \cdot\left(2,2 \mathrm{t} / \mathrm{m}^{3} \cdot 0,3 \mathrm{~m} \cdot 0,3 \mathrm{~m} \cdot 1,80 \mathrm{~m}\right)=0,712 \mathrm{t}$

Total de $\mathrm{P}=1,211 \mathrm{t}=\underline{1,211 \mathrm{kp}}$

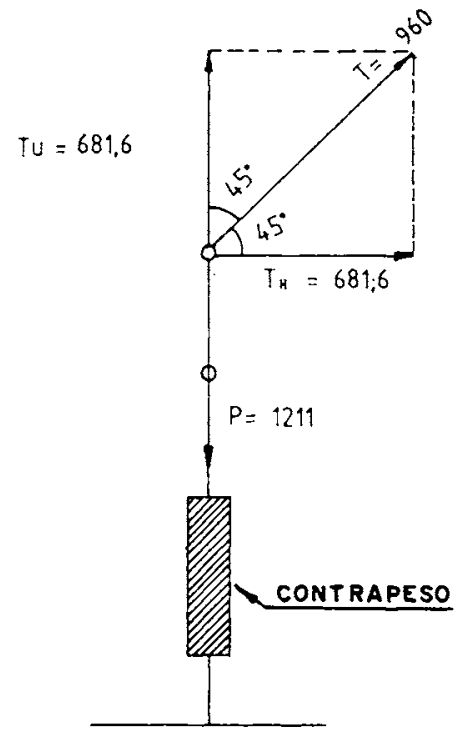




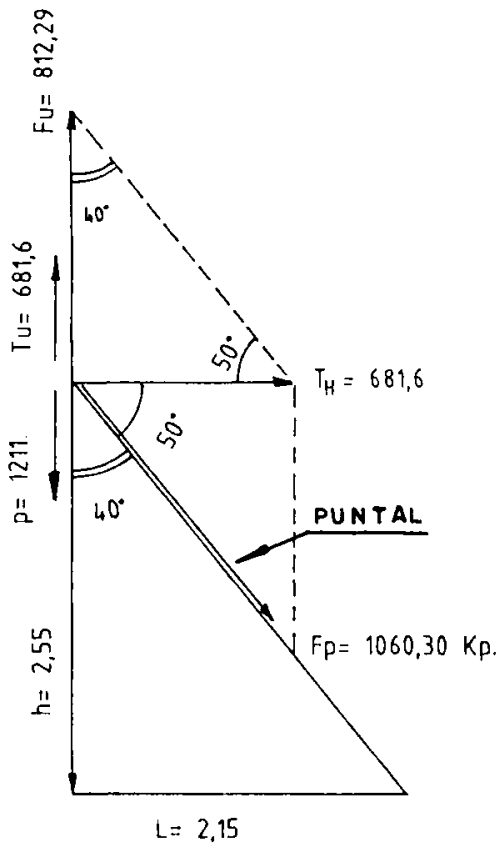

Descomponiendo la fuerza horizontal $T_{H}$ en la direccion del puntal y la dirección vertical, tenemos:

$F_{p}=781,6 \mathrm{~kg} / \cos 50=1060,30 \mathrm{~kg}$

$F_{v}=F_{p} \cdot \operatorname{sen} 50=812,29 \mathrm{~kg}$

Por lo tanto, la fuerza que tracciona el anclaje es de

$\Sigma F_{\mathrm{v}}=681,6 \mathrm{kp}+812,29 \mathrm{kp}-1211=282,9 \mathrm{kp}$

que se debe contrarrestrar con un peso suplementario (sacos de arena).

La fuerza $F_{p}$ de 1060,3 kp se contrarrestra con dos puntales según el esquema.

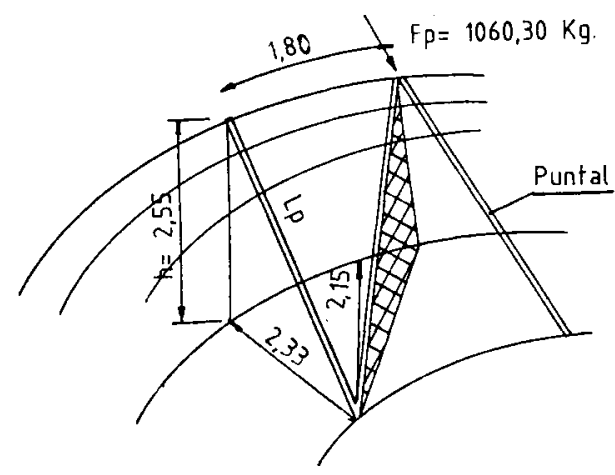

Para conocer la fuerza en cada puntal necesitamos conocer la longitud y el ángulo que forman entre sí. $1_{p}=2,55^{2}+2,33^{2}$

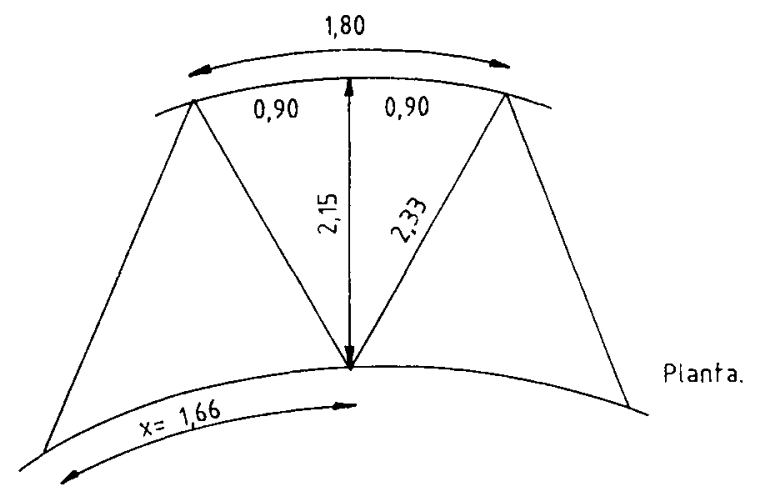

Por una relación de triángulos.

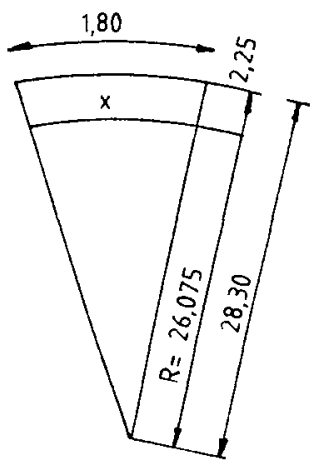

$x=26,075 \cdot 1,80 / 28,3$

$X=1,66 \mathrm{~m}$

Como $1 \cdot \operatorname{sen} \alpha=0,83=x / 2$

$\operatorname{sen} \alpha=0,83 / 3,45=0,25 \rightarrow \alpha=\operatorname{arcsen}(0,25) \rightarrow \alpha=14^{\circ}$

Por lo tanto la fuerza sobre cada puntal

$F_{\text {puntal }} \cdot \cos 14^{\circ}=1060,3 / 2$

$F_{\text {puntal }}=1060,3 /\left(2 \cdot \cos 14^{\circ}\right)=546 \mathrm{~kg}$ sobre el puntal.

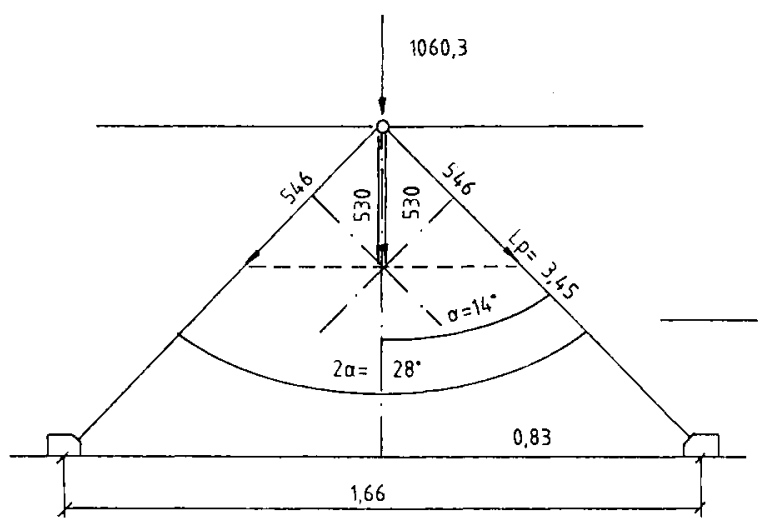




\section{CONFECCIÓN}

\section{Cálculo de los patrones de la esfera}

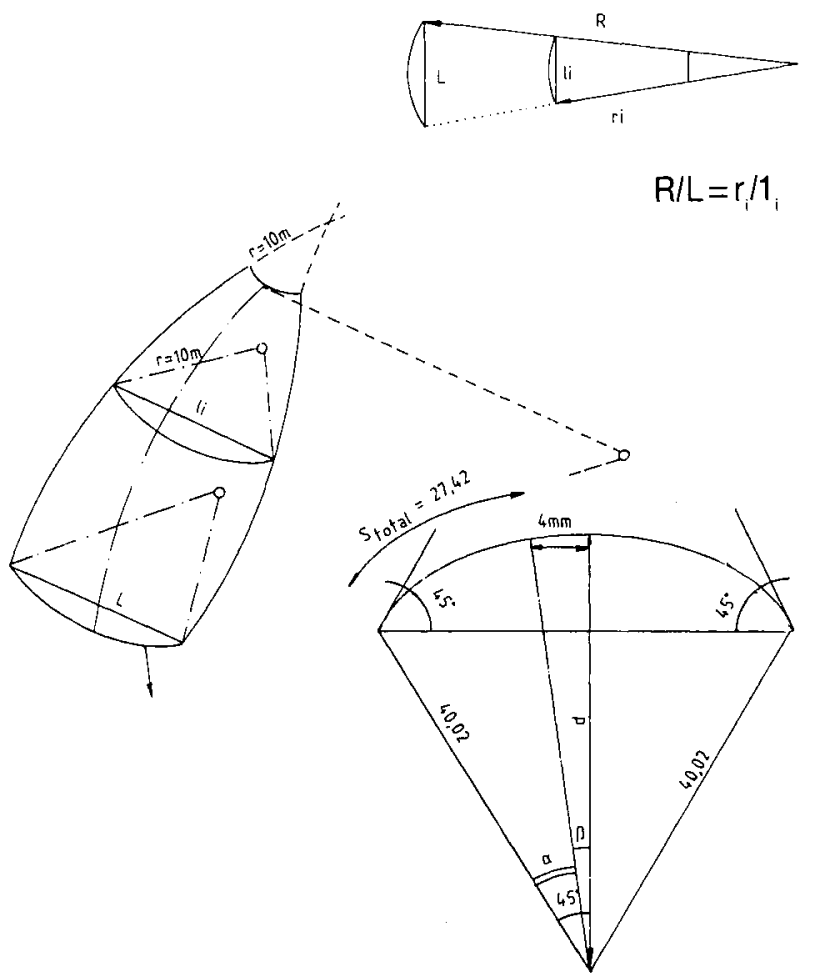

$\mathrm{d}^{2}+4^{2}=40,02^{2}$

$\mathrm{d}=40,02^{2} \cdot 16=39,82 \mathrm{~m}$

$\operatorname{tg} \beta=4 / d=4 / 39,82=0,100$

$\beta=\operatorname{arctg} 0,100=5,74$

$\alpha=45 \cdot 5,74=39,26$

$39,26 / \mathrm{s}=360 /(2 \cdot \pi \cdot 40,02) \quad \mathrm{s}=27,42 \mathrm{~m}$

$s / \alpha=s /(45-\beta)=2 \cdot \pi \cdot 40,02 / 360$

$360 \cdot s /(2 \cdot \pi \cdot 40,02)=45-\beta$

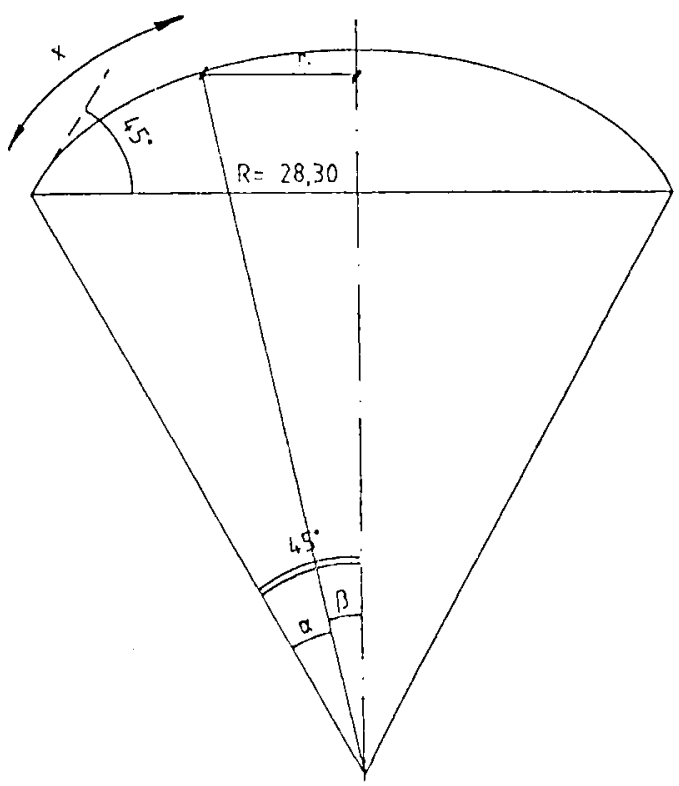

$\left.\beta=45-\left(\frac{360 * s}{2 * \pi * 40.02}\right)\right]$

$R * \operatorname{sen} \beta=r$

$\beta=\operatorname{arcsen} \frac{r}{R}$

$\operatorname{arcsen} \frac{r}{R}=45-\left(\frac{360 * s}{2 * \pi * 40.02}\right)$

$\frac{R}{L}=\frac{r}{I} ; \frac{R-i}{L}=r$

Para calcular el otro radio de curvatura de la tela $r=10 \mathrm{~m}$

$a_{o}$, depende de la anchura inicial del gajo de esfera; $L$, es la distancia entre dos puntos de anclaje medida en línea recta, que es el dato inicial de partida para cada gajo de esfera.

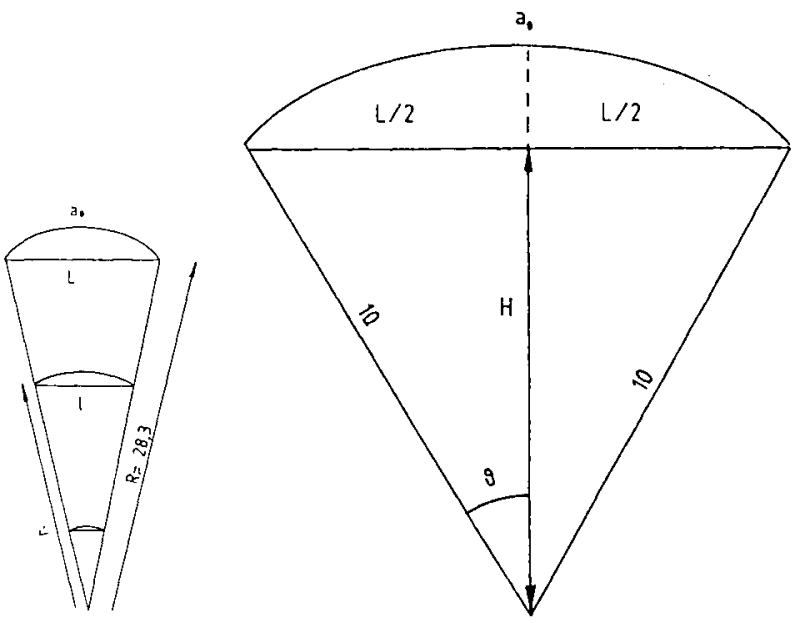

$$
\begin{aligned}
& 10 * \operatorname{sen} \theta=\frac{L}{2} \\
& \operatorname{sen} \theta=\frac{L / 2}{10} \quad \theta=\operatorname{arcsen} \frac{L / 2}{10} \\
& \frac{2 \sigma}{a_{0}}=\frac{360^{\circ}}{2 * \eta * 10} \quad \frac{2 * \operatorname{arcsen} L / 20}{a_{0}}=\frac{360^{\circ}}{2 * \eta * 10} \\
& a_{0}=\frac{4 * \pi * 10^{*} \operatorname{arcsen} L / 20}{360^{\circ}}
\end{aligned}
$$


Se deduce de igual manera la fórmula para calcular la relación entre cualquier otra longitud de circunfe. rencia y su arco correspondiente.

$$
I=20 * \operatorname{sen} \frac{a * 360}{40 * \pi} \quad \text { (3) }
$$

Sustituyendo (2) y (3) en (1)

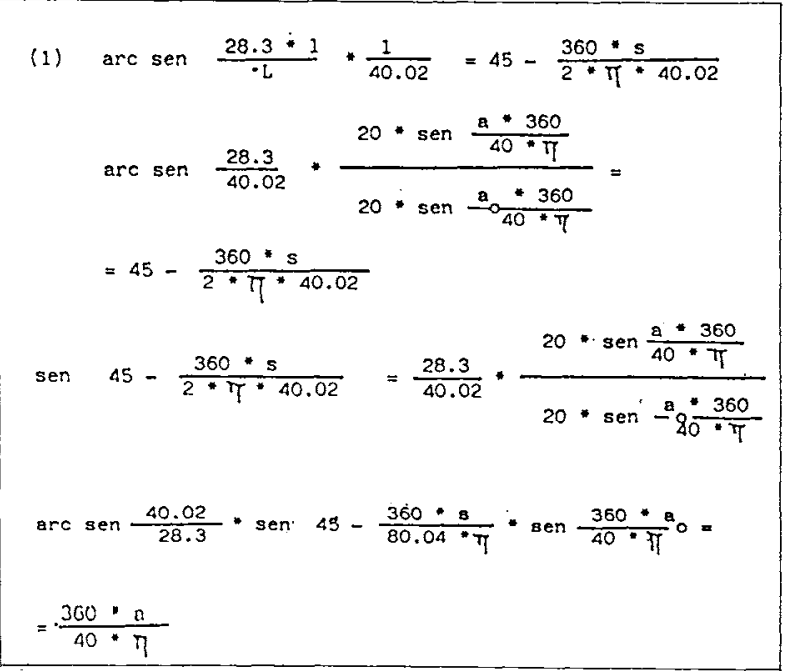

PROGRAMA PARA EL CÁCULO DE LOS PATRONES (Ordenador de bolsillo)

10 REM "Patrones"

20 PRINT "Cálculo del ancho del patrón cada $50 \mathrm{~cm}$ ".

30 INPUT "SEPARACIÓN ENTRE APOYOS = "; L

$40 \mathrm{AO}=0,3490658 \cdot \mathrm{ASN}(\mathrm{L} / 20)$

50 FOR $X=0$ TO 27,5 STEP 0,5

60 PRINT "X "," A ";," B"

70 IF $X=27,5$ THEN $X=27,42$

$80 \mathrm{~A}=0,34906585 \cdot \mathrm{ASN}[1,414134275 \cdot \operatorname{SIN}(45-1,4316786 \cdot X)$ $\cdot \operatorname{SIN}(2,864788975 \cdot A 0)]$

90 PRINT X;INT (1000·A)/1000; (3-(INT(1000·A)/1000))/2 100 IF $X=27,42$ THEN $X=27,5$

110 NEXT $X$

120 END

(c) Consejo Superior de Investigaciones Científicas Licencia Creative Commons 3.0 España (by-nc)

\section{EJEMPLO DEL CÁLCULO DEL PATRÓN}

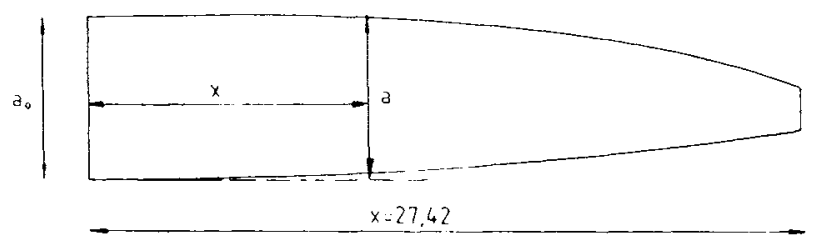

PATRÓN -A- $L=1,90 \mathrm{~cm}$

\begin{tabular}{lclc}
$x$ & $a(x)(m)$ & \multicolumn{1}{c}{$x$} & $a(x)(m)$ \\
0 & 1,4 & 14 & 1,13 \\
0,5 & 1,87 & 14,5 & 1,10 \\
1 & 1,85 & 15 & 1,07 \\
1,5 & 1,83 & 15,5 & 1,04 \\
2 & 1,87 & 16 & 1,01 \\
2,5 & 1,77 & 16,5 & 0,98 \\
3 & 1,75 & 17 & 0,95 \\
3,5 & 1,72 & 17,5 & 0,92 \\
4 & 1,70 & 18 & 0,88 \\
4,5 & 1,67 & 18,5 & 0,85 \\
5 & 1,65 & 19 & 0,82 \\
5,5 & 1,62 & 19,5 & 0,79 \\
6 & 1,59 & 20 & 0,76 \\
6,5 & 1,57 & 20,5 & 0,72 \\
7 & 1,54 & 21 & 0,69 \\
7,5 & 1,51 & 21,5 & 0,65 \\
8 & 1,48 & 22 & 0,63 \\
8,5 & 1,45 & 22,5 & 0,59 \\
9 & 1,43 & 23 & 0,56 \\
9,5 & 1,39 & 23,5 & 0,53 \\
10 & 1,37 & 24 & 0,49 \\
10,5 & 1,34 & 24,5 & 0,46 \\
11 & 1,31 & 25 & 0,43 \\
11,5 & 1,28 & 25,5 & 0,39 \\
12 & 1,25 & 26 & 0,36 \\
12,5 & 1,22 & 26,5 & 0,33 \\
13 & 1,19 & 27 & 0,29 \\
13,5 & 1,16 & 27,42 & 0,27
\end{tabular}

\section{CÁLCULO DEL VOLUMEN DE AIRE. VENTILADORES}

Hay que considerar dos renovaciones de aire por hora:

$$
V=1 / 3 \cdot \pi \cdot h^{2} \cdot(3 \cdot R-h)
$$
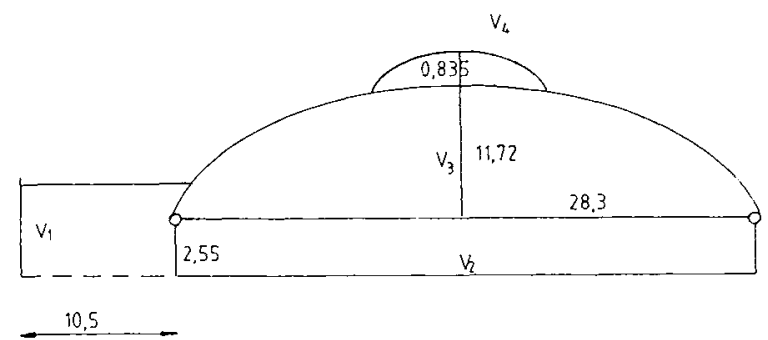
$V_{1}=2,55 \cdot 10,5 \cdot 3,20+\pi \cdot 1,5 \cdot 10,5=135,166 \mathrm{~m}^{3}$

$V_{2}=2,55 \cdot 2 \cdot \pi \cdot 20,3=453,43 \mathrm{~m}^{3}$

$V_{3}=1 / 3 \cdot \pi \cdot(11,72)^{2} \cdot(3 \cdot 28,3-11,72)=10526,3 \mathrm{~m}^{3}$

$V_{4}=1 / 3 \cdot \pi \cdot(0,835)^{2} \cdot(3 \cdot 4-0,835)=8,15193 \mathrm{~m}^{3}$

$V_{\text {total }}=11123 \mathrm{~m}^{3}\left(2 \cdot \mathrm{V}_{\text {total }}\right) /(1$ hora $)=22246 \mathrm{~m}^{3} /$ hora

$\mathrm{KW}$ necesarios $=$ Sup. en planta $\left(\mathrm{m}^{2}\right) / 250=$ $=\pi \cdot(28,3)^{2} / 250=10 \mathrm{~kW}$

Aplicando un coeficiente de seguridad de 3:

$\mathrm{Kw}$ necesarios $=30 \mathrm{Kw}$

\section{EJECUCIÓN}

Para la ejecución de la cúpula se debian cortar los patrones al tamaño adecuado obtenido en cálculo.

El replanteo se realizó extendiendo el plástico en el suelo y marcando las líneas de delimitación de los triángulos mixtilíneos (foto 5 ).

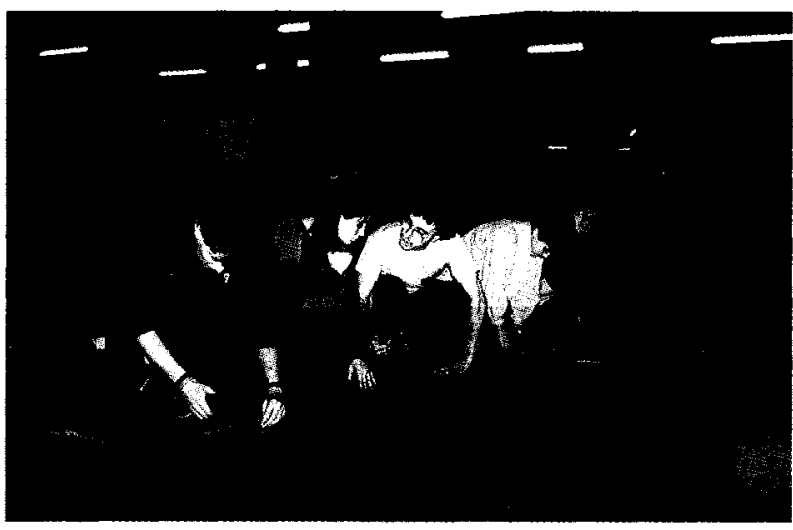

Foto 5

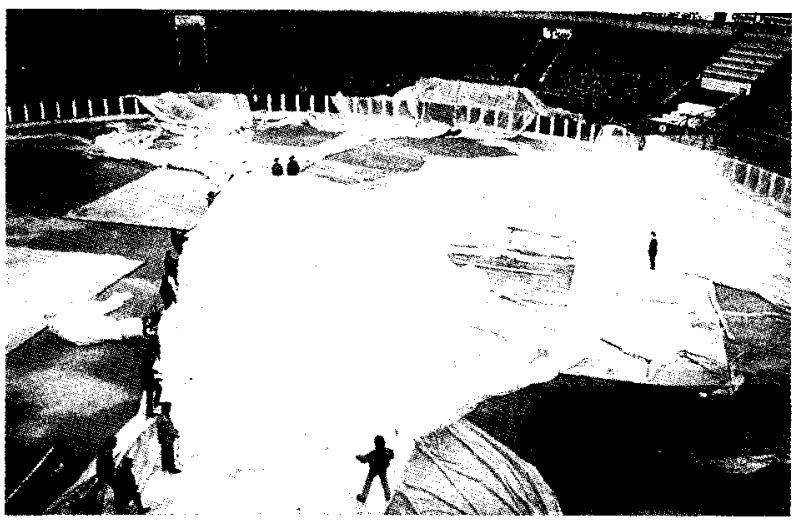

Foto 7
Cuando se iba a proceder al corte se hizo una simplificación consistente en no considerar la curvatura de las líneas longitudinales exteriores, error que tuvo que ser corregido posteriormente realizando unos patrones complementarios.

Obtenidos los patrones, se plegaron cuidadosamente y se transportaron a la plaza de toros, con el resto de útiles y herramientas necesarias (foto 6).

Una vez en la plaza, se fueron extendiendo los patrones y se procedió al cosido teniendo especial cuidado de mantener la linealidad de las uniones para evitar futuras arrugas (fotos 7 y $7^{\prime}$ ).

En el cosido se incluyó la cinta de refuerzo, doblando el plástico para la formación de la junta según se ha expuesto en el punto 2.

Se unieron todos los gajos de la cúpula de $\varnothing 56,45 \mathrm{~m}$ por un lado y el casquete esférico de remate por otro. Posteriormente se unieron ambos.

Una vez confeccionada la cúpula, hubo que deshacer parte de ella para introducir los patrones suplementarios ya comentados, para obtener una forma esférica, pues de lo contrario al haber simplificado los patrones, la superficie resultante hubiera sido cónica.

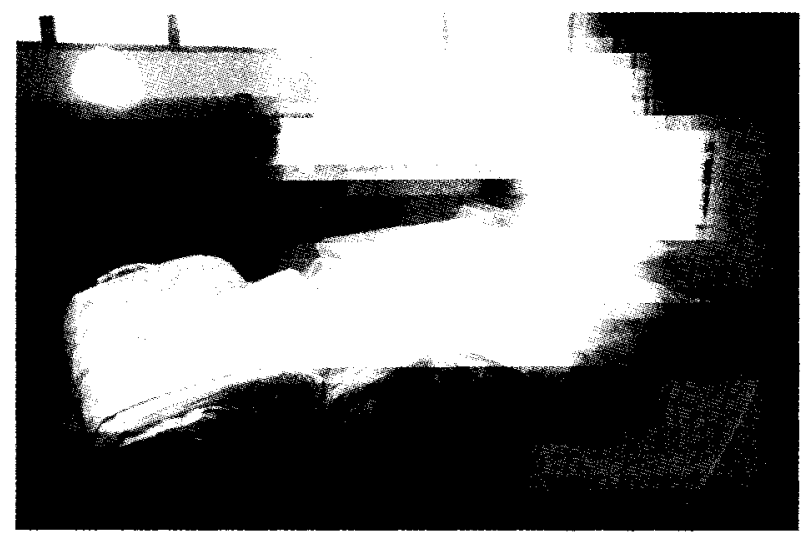

Foto 6

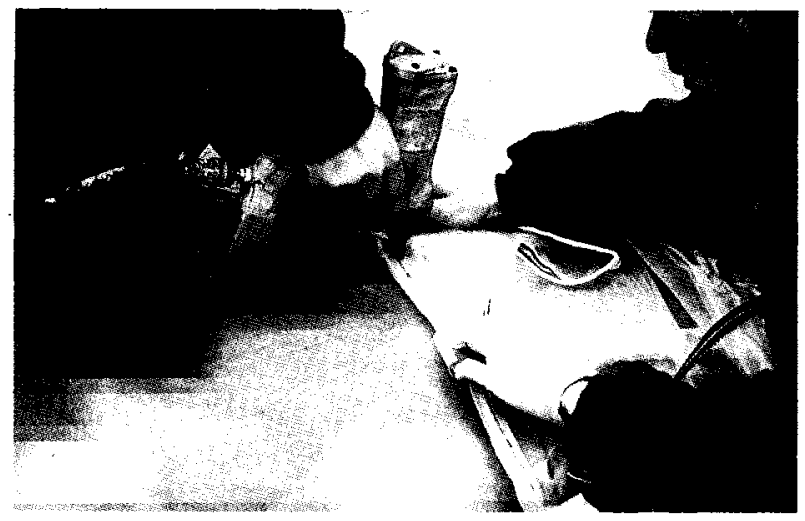

Foto 7 
La unión a la barandilla se realizó por medio de la cinta que previamente se había dejado sobrante al formar las juntas (fotos 8 y $8^{\prime}$ ).

Por otro lado y mientras se confeccionaban las uniones, se habia apuntalado la barandilla con ayuda de sacos terreros según se muestra en la foto 9 .

Finalmente se colocaron los faldones desde la barandilla al suelo para evitar las fugas de aire y se instalaron los ventiladores (foto 10).

Revisada toda la instalación se pusieron en marcha los ventiladores colocados perimetralmente con simetría radial, y el plástico comenzó a levantarse (fotos 11 a 17).

\section{CONSIDERACIONES FINALES}

\subsection{Intento de hinchado}

En este punto trataremos de analizar las causas que impidieron la elevación definitiva de la cúpula, aunque sólo sea como estimaciones.

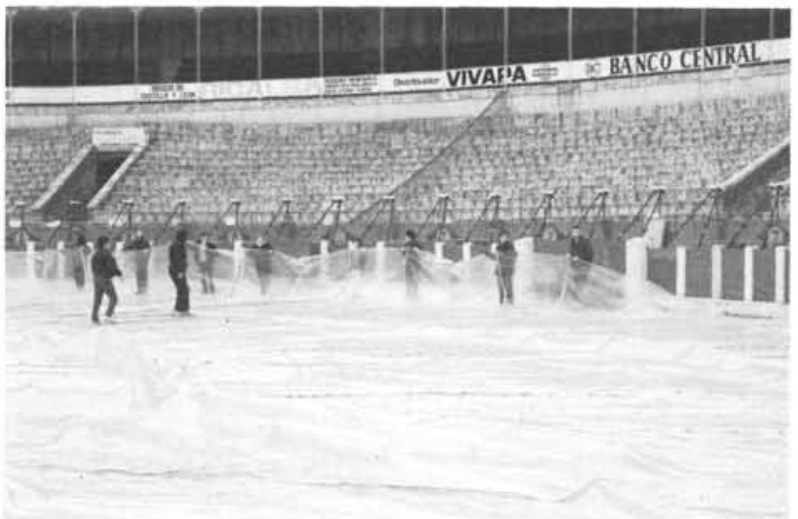

Foto 8

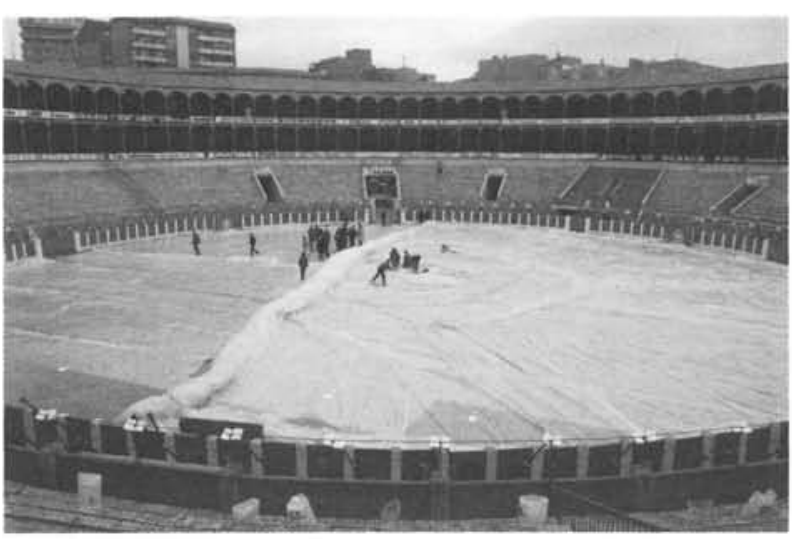

Foto 8
Los problemas se iniciaron en forma de nieve caída la noche anterior al dia acordado para levantar la estructura. El peso acumulado hizo imposible el intento y se decidió esperar a la jornada siguiente. Sin embargo, una lluvia posterior se sumó a la nieve derretida, haciendo necesaria la intervención de los bomberos para achicar el agua (foto 18).

Aún con todos estos inconvenientes, y debido al compromiso adquirido con los expositores, se intentó de nuevo el inflado, sin que las condiciones atmosféricas fueran todavia estables.

Los problemas de más difícil explicación aparecieron entonces. Por dos veces se intentó levantar la estruc. tura, pero resultaba imposible estabilizar el aire interior, que en forma de remolinos incontrolados chocaba contra los bordes con tanta fuerza que se rompian algunas juntas, remolinos provocados, probablemente, por el viento exterior.

Para evitar esto, los alumnos intentaron la estabilización de la cubierta de varios modos, entre ellos sujetando el centro de la cúpula, lugar donde se producian las mayores oscilaciones, colgándose de la misma, aunque sin conseguir evitar los torbellinos.

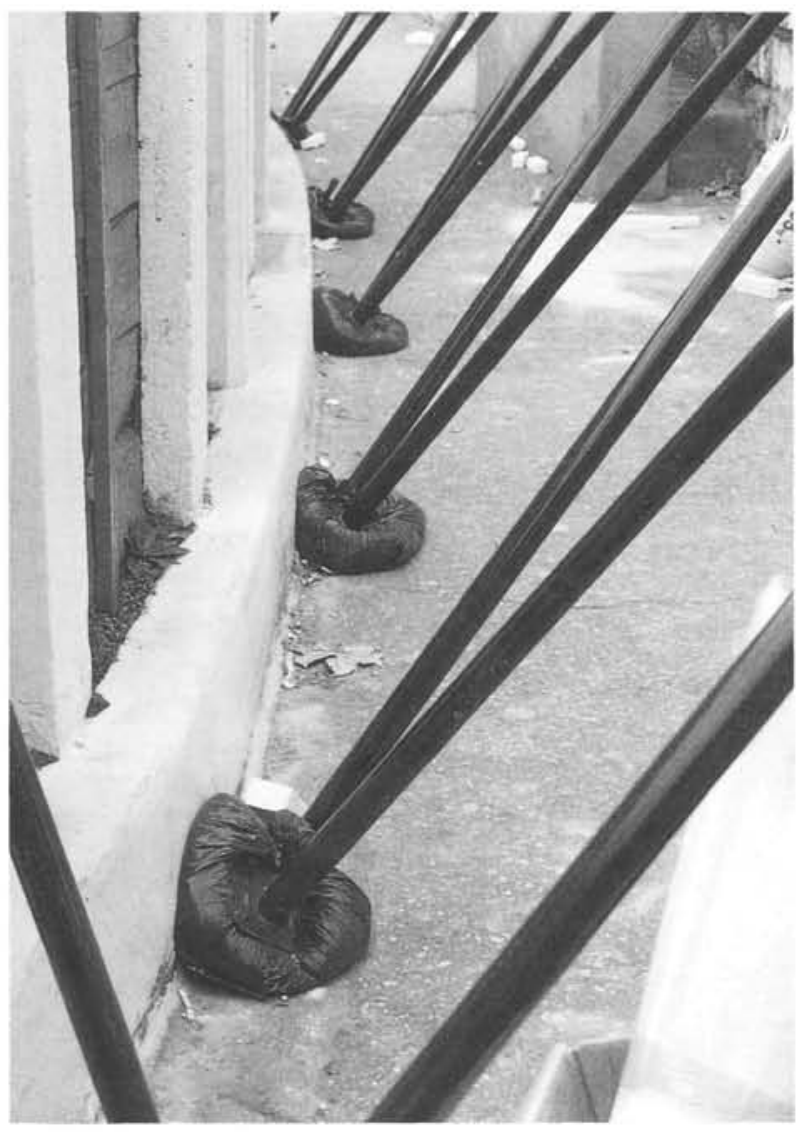

Foto 9 


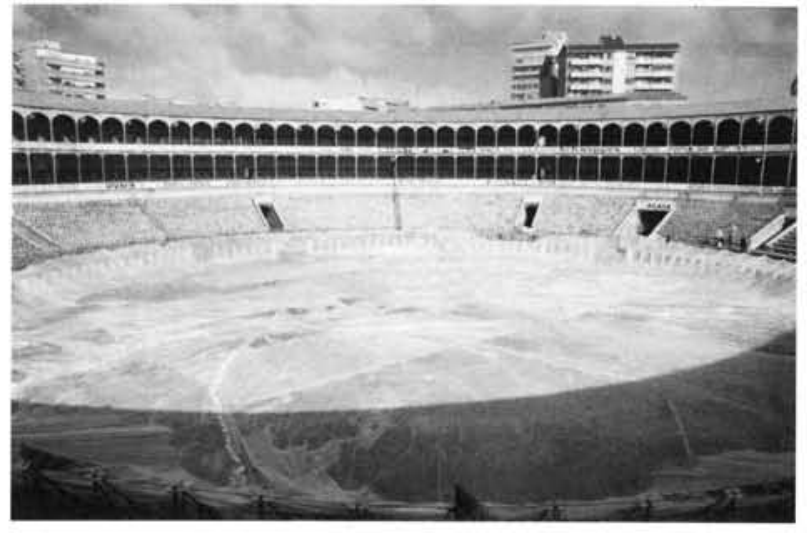

Foto 10

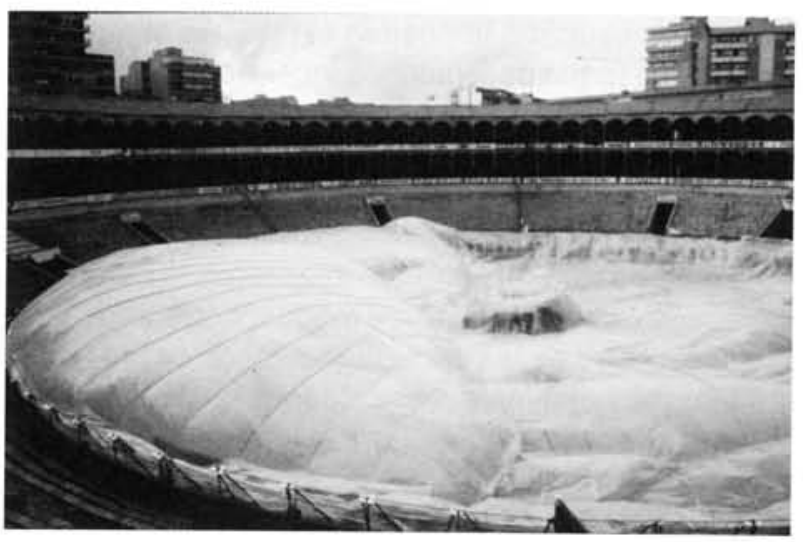

Foto 12

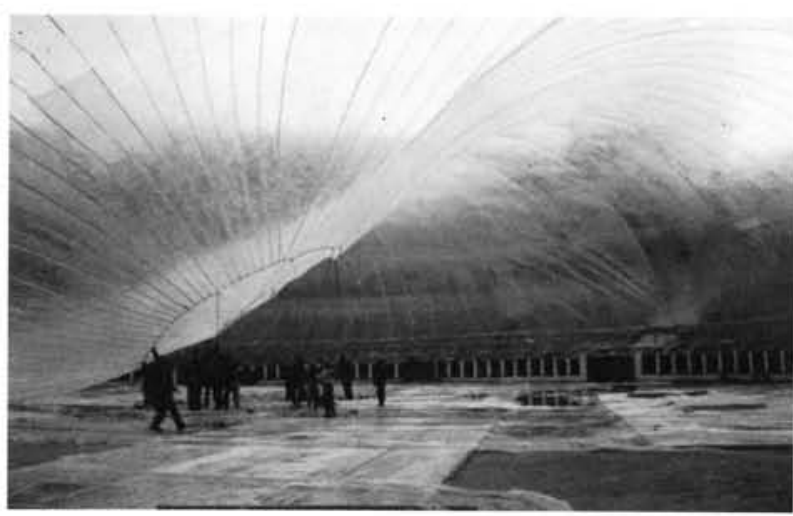

Foto 14

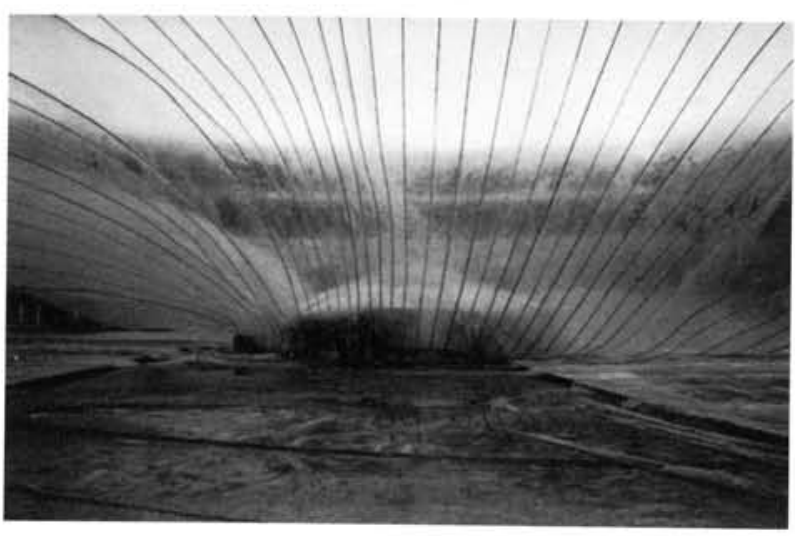

Foto 16

(c) Consejo Superior de Investigaciones Científicas Licencia Creative Commons 3.0 España (by-nc)

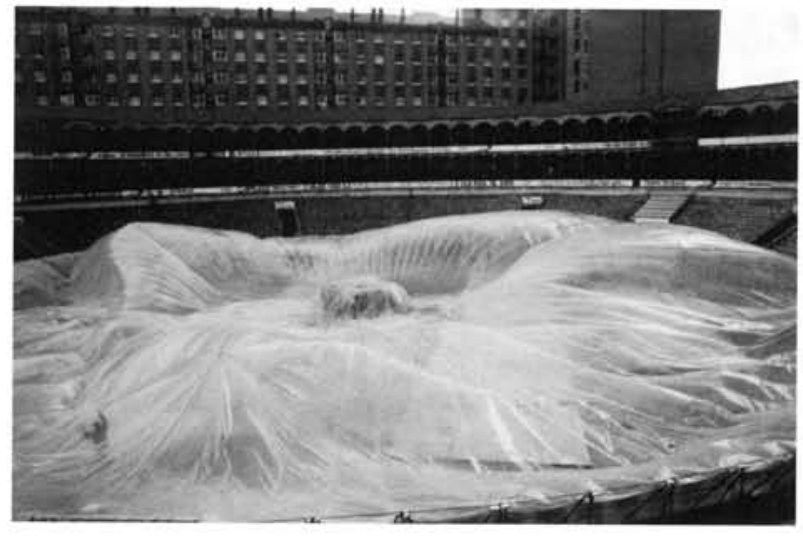

Foto 11

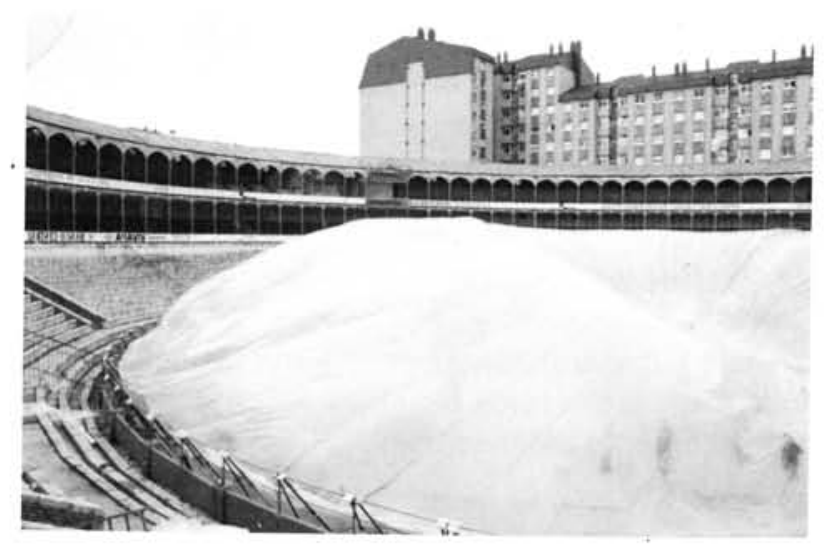

Foto 13

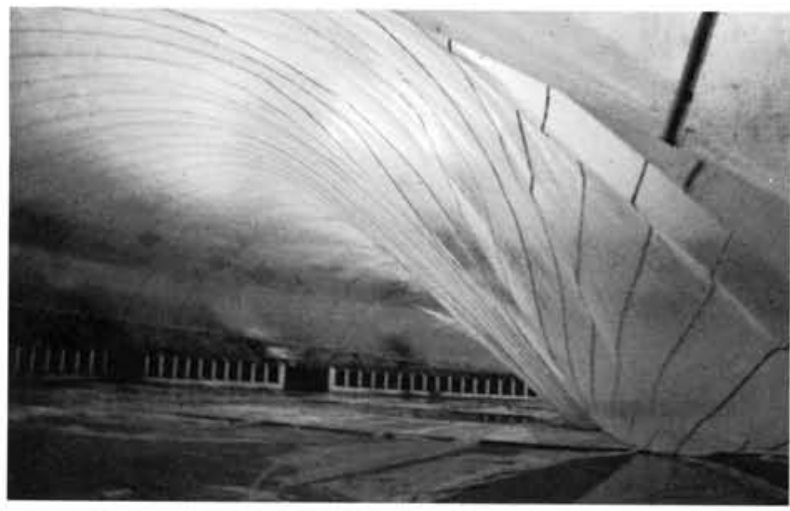

Foto 15

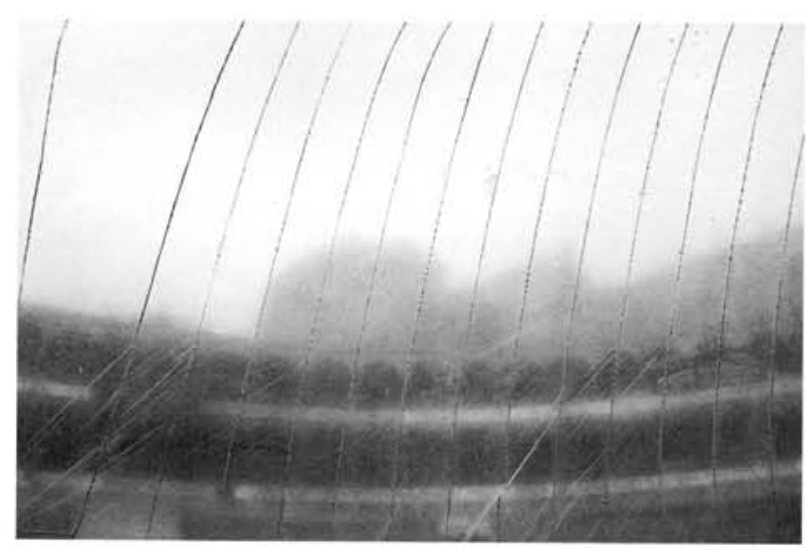

Foto 17

http://informesdelaconstruccion.revistas.csic.es 
Como no resultaba posible terminar el hinchado, se decidió de nuevo suspender el proceso hasta que las condiciones atmosféricas mejorasen (foto 19).

La cúpula se bajó definitivamente, quedando anclada sólo por su perímetro hasta el dia siguiente, pero los vientos que soplaron esa noche fueron huracanados y los remolinos que se formaron en la plaza destrozaron poco a poco la cubierta.

\subsection{Breve análisis del problema}

Analizando las condiciones de hinchado, podemos deducir lo siguiente:

Los ventiladores, tres de $5 \mathrm{CV}$ y tres de $2 \mathrm{CV}$, situados en radios de $60^{\circ}$, encontraron dificultades con el burladero que bordea la plaza y su teórica inclinación de $45^{\circ}$ resultaba en la práctica una proyección de aire directa contra la membrana, cuando la impulsión debería haberse realizado hacia el centro e iniciarse en el mismo.

Los golpes producidos contra los bordes por el movimiento ondulatorio generado en la tela, se producian a media hora de iniciado el hinchado, habiéndose calculado unos cuarenta minutos para el hinchado total.

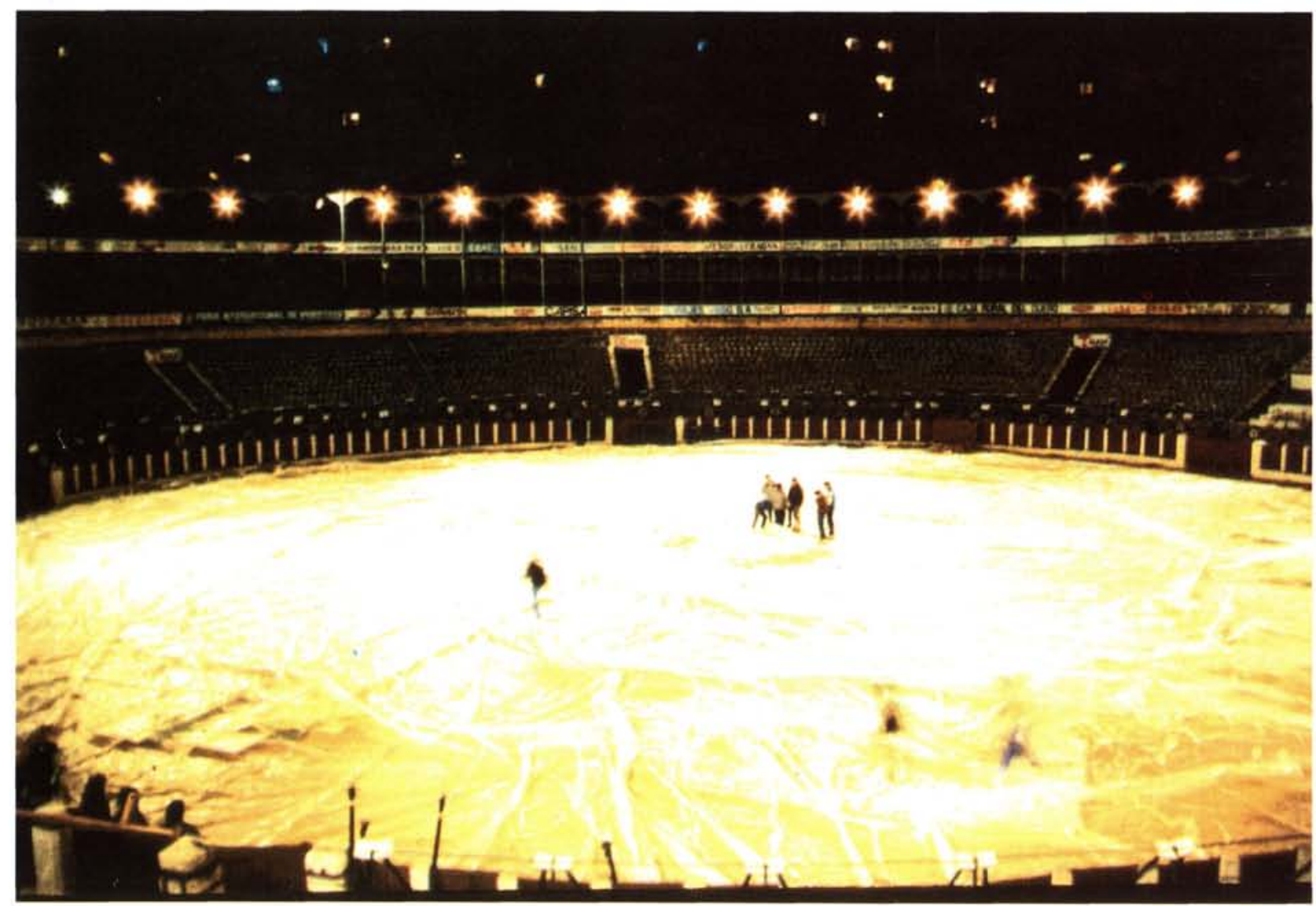


Hay que tener en cuenta que mientras se levanta la membrana deben inspeccionarse las costuras, cables, anclajes, etc. por lo que no es recomendable efectuar el proceso más rápidamente, de la misma forma que hacerlo demasiado lento tampoco es aconsejable, pues pueden producirse variaciones importantes de las condiciones climáticas exteriores, con posible aparición de vientos, lluvias, etcétera.

Quizás hubiera sido recomendable haber realizado además el hinchado con aire calefactado y aprovechar así su menor densidad, de forma semejante al inflado de globos aerostáticos, sobre todo teniendo en cuenta que los intentos se hicieron al atardecery en el mes de abril.

De cualquier forma las condiciones exteriores con viento excesivo parecian condenar el proyecto. Creemos que se debió retrasar la elevación hasta que las condiciones atmosféricas fuesen de la mayor calma posible, ya que este tipo de cubiertas sólo son estables al viento cuando están hinchadas, pues sólo con la tensión se resiste la presión exterior.

Por otra parte, en esas condiciones, podía resultar especialmente desfavorable la forma geométrica de la burbuja de gran planeidad.

Por último, y quizás más importante, sea decir que la baja calidad del plástico empleado, no hay que olvidar que se trataba de un proyecto experimental, contribuyó de forma considerable a su mal comportamiento. El envejecimiento acelerado a que había sido sometido por el hielo y el agua disminuyó al mínimo las condiciones elásticas, haciéndolo romper fácilmente.

\section{publicación del ICCET/CSIC}

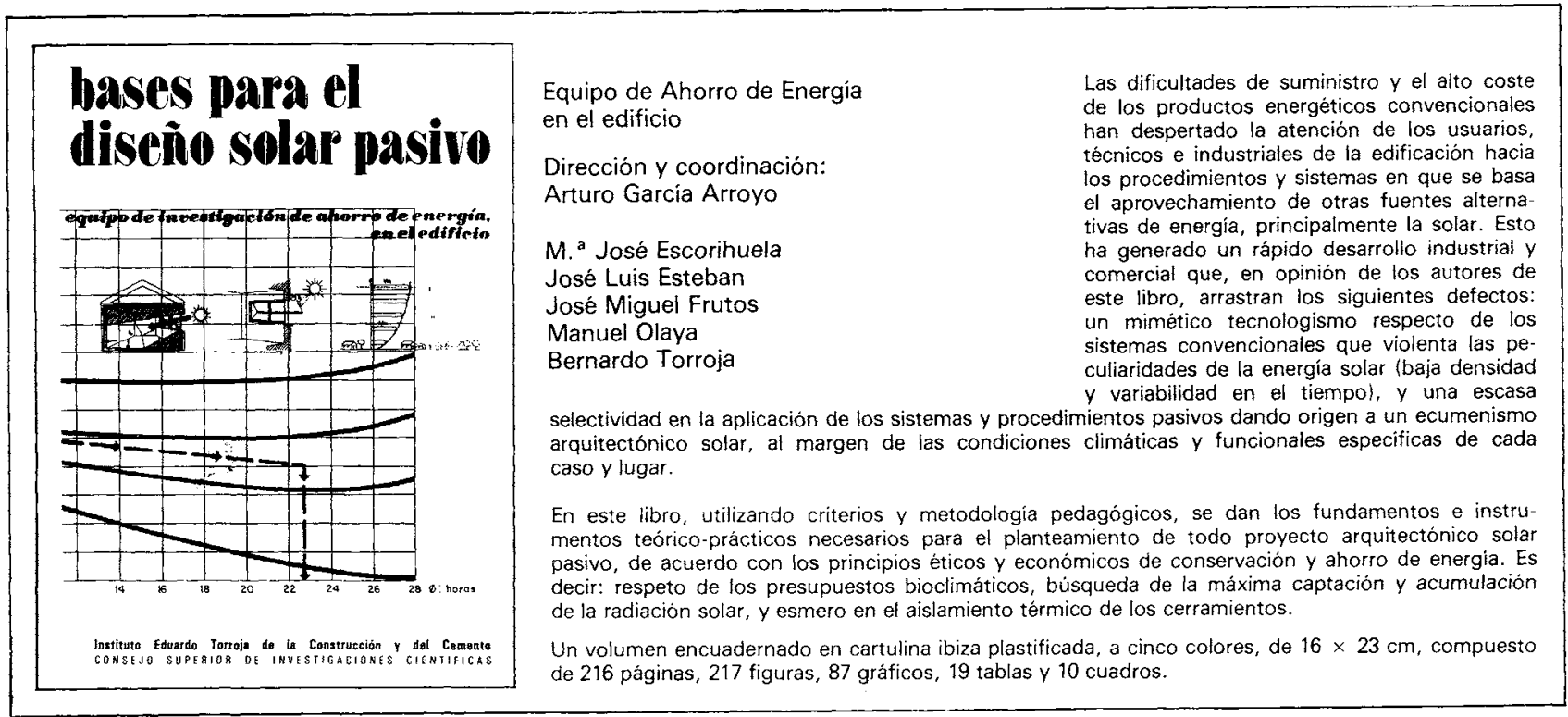

